\section{More confident trauma resuscitation team leaders: a novel simulation-based training curriculum utilizing video feedback}

\author{
John L. Falcone,' Paul E. Phrampus,' \\ Andrew B. Peitzman, ${ }^{1}$ Louis H. Alarcon, ${ }^{1}$ \\ Raquel M. Forsythe ${ }^{1}$ \\ 'University of Pittsburgh School of \\ Medicine, University of Pittsburgh \\ Medical Center, Department of Surgery, \\ Division of Trauma and Acute Care \\ Surgery, Pittsburgh, PA; ${ }^{2}$ University of \\ Pittsburgh School of Medicine, University \\ of Pittsburgh Medical Center, Department \\ of Emergency Medicine, Pittsburgh, PA, \\ USA
}

\section{Abstract}

There are deficiencies in trauma leader performance. Simulation training and videobased feedback can lead to durable changes in behavior. A trauma resuscitation team leader training curriculum was developed. The curriculum consisted of eight simulated trauma scenarios with a mix of acuities and injury patterns using patient simulators. Other team members included a surgeon, an anesthesiologist, a chief resident, a trauma nurse, a medical student, and presenting emergency medicine staff. Each scenario was followed by video-based feedback. Attitudes regarding this curriculum were evaluated before and after the intervention with Likert-based surveys. Eight residents completed the curriculum. On a seven-point Likert scale, the median overall curriculum rating, the video discussion quality, the plan to apply leadership skills, and the plan to apply learned knowledge and behaviors was 7/7. A Wilcoxon Sign-Rank test showed improved confidence for leading Level 1 trauma resuscitations, improved beliefs in adequate training, and improved attitudes regarding team leader training $(\mathrm{P}<0.05)$. There was reduced nervousness of being the team leader $(\mathrm{P}=0.048)$. Qualitative analyses showed that the learners valued the feedback process and scenario realism. This pilot curriculum was well-received by trauma residents and offers insight into meta-cognition of trauma team leaders.

\section{Background}

The trauma resuscitation team leader is pri- marily responsible for conducting the initial assessment and survey, coordinating and directing team and individual activities, and formulating plans for definitive management of acutely-injured patients. ${ }^{1}$ There are deficiencies in trauma resuscitation leader performance in two domains: the domain of Advanced Trauma Life Support (ATLS) adherence and the domain of other leadership behaviors. $^{2-5}$

There is potential to improve trauma resuscitation leadership behaviors with video review and feedback. ATLS-based video review and feedback can lead to shorter and more efficient trauma resuscitations. ${ }^{6}$ Video-based review leads to rapid and sustained learning for trauma leaders, as it is more effective than verbal feedback in achieving behavioral changes and algorithm compliance. ${ }^{7}$

The use of human patient simulation engages emotional and psychomotor learning while fostering critical thought processes, communication, and interaction. ${ }^{8}$ ATLS training has led to improved trauma leader performance in simulation exercises. ${ }^{9}$ As a corollary, instructor-based learning may be more effective than self-directed learning for some aspects of learning during a clinical simulated experience. ${ }^{10}$ At the University of Pittsburgh Medical Center (UPMC) Level 1 Trauma Center, approximately 5000 acutely injured patients are evaluated annually. The trauma resuscitation team leader is typically a resident at the post-graduate year two or three level in surgery or emergency medicine. There is currently no formal method to train trauma resuscitation leaders during the trauma rotation. Trauma resuscitation team leader training is done with ATLS certification of all trauma residents, as well as weekly video-based discussion of Level 1 trauma resuscitations.

With the deficiencies in leadership behavior, the benefit of ATLS-based video review, and the benefit of simulation training, we believe that a dedicated trauma resuscitation team leader training curriculum would benefit our trauma resuscitation team leaders. We developed a new curriculum to train trauma resuscitation leaders in the ATLS and leadership domains. This is the first description of a specific trauma team leader training curriculum. It addresses specific needs based on team leader performance, and uses high-fidelity simulation for training that has high-stakes impact for patients.

\section{Innovation}

\section{Curriculum study}

In this prospective curriculum evaluation, trauma resuscitation leaders were block ran-
Correspondence: John L. Falcone, University of Pittsburgh Medical Center, Presbyterian University Hospital, F-675, 200 Lothrop Street, Pittsburgh, PA 15213, USA.

Tel. +1.412.6472345 - Fax: +1.412.6474889

E-mail: falconej@upmc.edu

Key words: competency-based education, curriculum, educational measurement, interdisciplinary health team, patient simulation.

Conflict of interests: the authors declare no potential conflict of interests.

Acknowledgements: the authors would thank all of the members of the Peter M. Winter Institute for Simulation Education and Research for their contribution to curriculum development and implementation.

Received for publication: 1 September 2012.

Revision received: 12 December 2012.

Accepted for publication: 14 January 2013.

This work is licensed under a Creative Commons Attribution NonCommercial 3.0 License (CC BYNC 3.0).

(C) Copyright J.L. Falcone et al., 2013

Licensee PAGEPress srl, Italy

Medical Education Development 2013; 3:e1

doi:10.4081/med.2012.e1

domized 1:1 by specialty (surgery or emergency medicine) and by post-graduate year (two or three) over six months. Randomization occurred with a random number generator. This pilot curriculum study was approved by the Institutional Review Board.

\section{Curriculum intervention}

The overview to this curriculum is given in Figure 1. After recruitment, residents electronically received pre-reading material. This material included the goals and objectives for the curriculum. These goals and objectives were generated as a result of a modified Delphi technique between trauma surgeon faculty (LA, RF), emergency medicine faculty (PP), and a surgery resident trained in medical education (JF). These goals and objectives are listed in Table 1. As a supplement to the goals and objectives, the pre-reading material also included two electronic chapters from a primary text used by the trauma service regarding organization prior to trauma patient arrival and adult trauma resuscitation (19 pages).,11

The curriculum was given at the start of the trauma rotation at the Peter M. Winter Institute for Simulation Education and Research (WISER) in Pittsburgh, Pennsylvania. Resident team leaders first electronically filled out a pre-curriculum survey including demographic information and attitu- 
dinal assessments of trauma team leadership and simulation training (5 minutes). This was followed by a one-on-one didactic session with a trauma surgeon (RF) (20 minutes). In this didactic session, the curriculum schedule was reviewed. Curriculum goals and objectives were individually addressed. A trauma resuscitation video demonstrating favorable team leader behaviors was critiqued in a similar fashion to subsequent critiques of the resident team leader (10 minutes). The resident team leader was then oriented to the simulated trauma bay, including the manikins and their capabilities and limitations, and to the other members of the trauma team (10 minutes).

Other members of the trauma resuscitation team included a trauma surgeon (RF), a chief resident (JF), a trauma intern, EMS personnel, trauma nurses, medical students, and an anesthesiologist. Volunteers were used for these roles, and included EMS volunteers, trauma nurse volunteers, and medical student volunteers.

Table 1. Goals and objectives for a trauma resuscitation team leader training curriculum.

Improve resuscitation leader adherence
to ANUS protocol: guidelines
1. Primary survey
Airway
Breathing
Circulation
Disability
Exposure
2. Secondary survey
Head
Neck
Chest
Abdomen
Pelvis
Upper extremities
Lower extremities
FAST
3. Decision-making algorithms
Improve other performance behaviors of
trauma resuscitation: leaders

1. Anticipation of and planning for potential problems

2. Assumption of leadership role

3. Communication with other team members

4. Distribution of workload/delegation of responsibility

5. Attention allocation

6. Utilization of information

7. Recognition of limitations/call for help early enough

8. Professional behavior/interpersonal skills

ATLS, advanced trauma life support

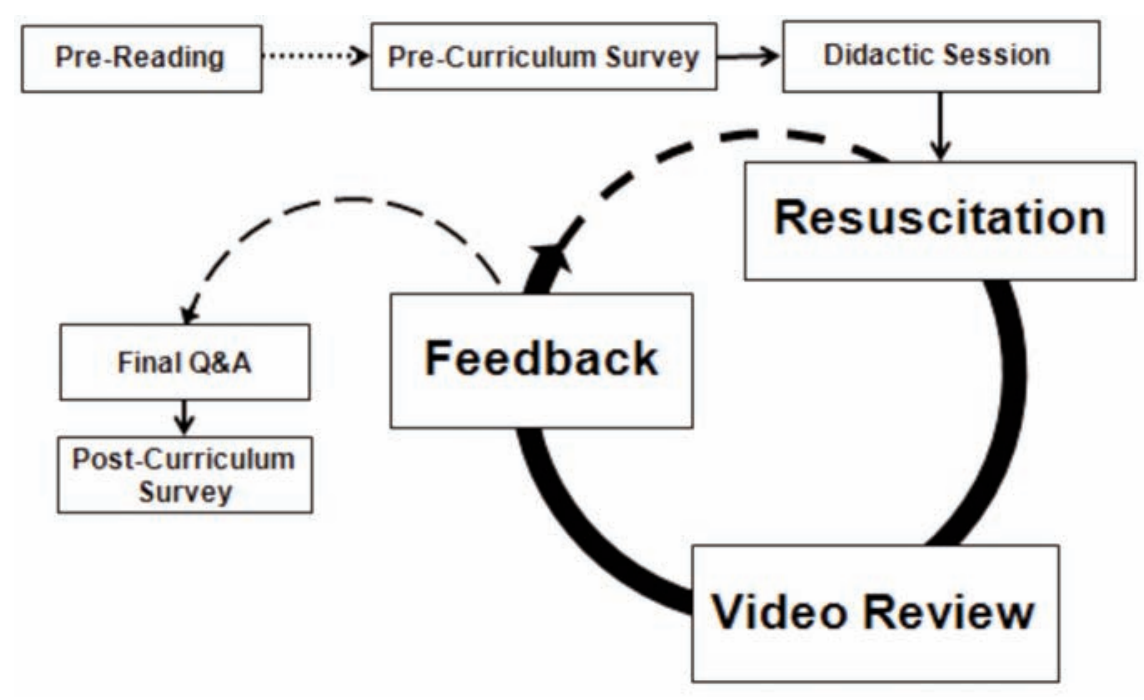

Figure 1. Overview of a trauma resuscitation team leader training curriculum.

Table 2. Demographics for eight trauma residents taking a dedicated trauma resuscitation leader training curriculum.

\begin{tabular}{lc} 
Characteristics* & \\
Age [Median, (IQR)] & $28.0(26.0-29.5)$ \\
Sex & $5(62 \%)$ \\
$\quad$ Female & $3(38 \%)$ \\
$\quad$ Male & $2(35 \%)$ \\
\hline Post-graduate year level & $6(75 \%)$ \\
$\quad$ Two & \\
$\quad$ Three & $4(50 \%)$ \\
Residency & $4(50 \%)$ \\
$\quad$ Surgery & $8(100 \%)$ \\
Emergency Medicine & $8(100 \%)$ \\
Certified in basic life support & $8(100 \%)$ \\
Certified in advanced trauma life support & $5.5(5.0-6.0)$ \\
\hline Acted as a trauma team leader before curriculum & \\
Being a trauma leader is appropriate for my level of training & \\
(7-point Likert Scale) [Median, (IQR)] &
\end{tabular}

IQR, inter-quartile range. *Characteristics are given as [n, (\%)] unless otherwise given.

Table 3. Evaluation of a human patient simulator-based trauma resuscitation team leader training curriculum using a 7-point Likert Scale.

\begin{tabular}{lc}
\hline Survey item & $\begin{array}{c}\text { Evaluation } \\
\text { [median,(IQR)] }\end{array}$ \\
Overall curriculum rating & $7.0(6.5-7.0)$ \\
The objectives of the course were clearly stated & $6.5(6.0-7.0)$ \\
\hline The course was appropriate for my level of training & $7.0(6.0-7.0)$ \\
The simulation scenarios were realistic & $6.5(5.0-7.0)$ \\
\hline The discussion of my performance was helpful & $7.0(6.0-7.0)$ \\
The debriefing was completed in a professional, non-personally threatening manner & $7.0(6.0-7.0)$ \\
\hline The debriefing experience allowed me to learn from my mistakes & $6.5(6.0-7.0)$ \\
I plan to apply what I learned here to my practice (knowledge, skills) & $7.0(6.0-7.0)$ \\
\hline I would recommend that this course be a part of the training of every trauma resident & $6.0(6.0-7.0)$ \\
\hline IQR, inter-quartile range. &
\end{tabular}


Table 4. Changes in eight trauma resuscitation leader attitudes after a dedicated trauma resuscitation leader training curriculum using a 7-point Likert Scale.

\begin{tabular}{|c|c|c|c|}
\hline Survey item & $\begin{array}{l}\text { Pre-curriculum } \\
\text { [Median, (IQR)] }\end{array}$ & $\begin{array}{l}\text { Post-curriculum } \\
\text { [Median, (IQR)] }\end{array}$ & P value* \\
\hline I feel confident in my ability to be the trauma resuscitation leader for Level 2 traumas & $6.0(5.5-6.0)$ & $6.0(6.0-7.0)$ & 0.18 \\
\hline I feel confident in my ability to be the trauma resuscitation leader for Level 1 traumas & $5.0(3.0-5.0)$ & $6.0(5.0-6.5)$ & 0.01 \\
\hline I have been trained adequately to be a trauma resuscitation leader & $5.0(4.5-5.0)$ & $6.0(5.5-6.0)$ & 0.01 \\
\hline I think dedicated team leader training is valuable & $7.0(5.5-7.0)$ & $6.0(6.0-7.0)$ & 0.65 \\
\hline I think simulation team leader training is valuable & $6.0(5.5-6.0)$ & $6.5(6.0-7.0)$ & 0.047 \\
\hline I feel nervous about being a team leader & $5.0(4.0-5.0)$ & $3.0(3.0-5.0)$ & 0.048 \\
\hline
\end{tabular}

$\mathrm{IQR}$, inter-quartile range. *P values for testing differences in pre-curriculum and post-curriculum item medians with a Wilcoxon Signed-Rank test.

Table 5. Trauma resuscitation leadership curriculum feedback by four participants.

Elements of the curriculum that were enjoyed

Feedback and video.

Simulation reviews.

I enjoyed the simultations [sic], the one on one direction froma trauma attending, the effort to make it feel as real as possible.

The scenarios were realistic and helped improve my team leader skills. I enjoyed the debriefings, and feel that my skills improved.
Elements of the curriculum that needed improvement None.

Need more situations where the team leader needs to delegate tasks (putting in chest tubes, placing IVs, reading films, calling services, etc.) while paying attention to the overall plan of the patient.

More feedback for communication, more working thru [sic] scenarios, more asking what the resident should be doing at that point, being very critical in terms of all the decision making process. More leader building with specific comments and specific suggestions.

It should be available to all residents.
The majority of the curriculum revolved around a series of simulated trauma patient scenarios. Eight case scenarios were written by a trauma surgeon (RF) and a surgery resident trained in medical education (JF). Cases were programmed by a WISER simulation specialist (NE) with time-specific decision-trees using Simman 3g software (Laerdal Medical, Wappingers Falls, NY). The scenarios were pilot-tested and revised. The cases were a mix of six blunt (75\%) and two penetrating (25\%) mechanisms. The cases represented a mix of Level 1 (higher) and Level 2 (lower) acuities. The resident team leader was presented with pre-hospital information through alphanumeric text pages. The resident team leader was the trauma resuscitation team leader for these eight patient scenarios (5-10 minutes per scenario). After each scenario, the resuscitation video was reviewed in a one-on-one fashion with the trauma surgeon (RF). Personalized feedback was given regarding the resident team leader performance, using the curriculum goals and objectives as a standard reference (10-15 minutes per feedback session).

After the eighth scenario, a final feedback wrap-up session occurred (10 minutes). Finally, the resident team leaders electronically filled out a post-curriculum survey including curriculum assessment and attitudinal assessments of trauma team leadership and simulation training (5 minutes).

\section{Evaluation methods}

Descriptive statistics were performed for all of the items assessed on the pre-curriculum and post-curriculum surveys. One-tailed Wilcoxon Signed-Rank tests using matched pairs were performed on identical items on both the pre-curriculum and post-curriculum surveys to test for differences in attitudes. Qualitative thematic analyses of elements of the curriculum that were enjoyed and elements of the curriculum that needed improvement were conducted for post-curriculum feedback. All statistical tests were performed with Stata 11.1 statistical software (StataCorp, College Station, Texas), using an $\alpha=0.05$.

\section{Evaluation}

Over the six-month study period, there were 18 resident team leaders that rotated on the trauma service. At the post-graduate year three level, 2/4 surgery residents and 4/9 emergency medicine residents were block randomized to receive the curriculum. At the post-graduate year two level, 2/5 surgery residents were selected. Demographics of the resident team leaders are given in Table 2 . There are no differences in the participant sex $(\mathrm{P}=0.73)$, postgraduate year level $(\mathrm{P}=0.29)$, or residency type $(\mathrm{P}>0.99)$. All participants were certified in Basic Life Support (BLS), ATLS, and had participated as the trauma resuscitation team leader prior to the curriculum.

The post-curriculum survey evaluation on a 7 -point Likert scale is given in Table 3 . The median overall curriculum rating, concordance of the curriculum with training level, discussion of performance, the nature of the individual feedback, and the plan to apply the knowledge and skills of the curriculum was 7/7.

The comparisons of pre-curriculum and post-curriculum attitudes are given in Table 4. Resident team leaders felt more confident for higher-acuity trauma resuscitation performance $(\mathrm{P}=0.01)$, adequately trained after the curriculum $(\mathrm{P}=0.01)$, with improved attitudes in team leader training $(\mathrm{P}=0.047)$ and reduced nervousness about team leadership $(\mathrm{P}=0.048)$.

A total of $4 / 8$ (50\%) participants offered feedback for qualitative thematic analyses. All $4 / 4$ (100\%) gave both elements of the curriculum that were enjoyed and elements of the curriculum that needed improvement. All of the typed feedback offered about this trauma resuscitation leader curriculum is shown in Table 5. Qualitative thematic analyses showed that a majority of elements enjoyed by the participants included the realism of the scenarios and the nature of the video-based feedback. A majority of the participants felt that more chances for feedback should have occurred in this curriculum. 
M, Schwab CW, et al, eds. The trauma manual: trauma and acute care surgery, $3^{\text {rd }}$ Ed. Philadelphia, PA: Lippincott Williams \& Wilkins; 2008. pp 62-70.

We successfully developed a needs-assessment-based and well-received simulation curriculum for trauma team leaders. Despite the small number of learners and low study power, this pilot curriculum leads to significantly improved attitudes and self-evaluation outcomes by junior residents. Similar applications to other team-based scenarios in other medical domains are possible. This is the first known study to describe the attitudes, self-evaluation, and meta-cognition of trauma resuscitation team leaders. This curriculum will be used to train future trauma residents. Furthermore, we plan to continue to evaluate curriculum outcomes, with the ultimate goal of evaluating team leader behaviors in live trauma bay resuscitations.

\section{References}

1. Hoff WS. Organization prior to trauma patient arrival. In: Peitzman AB, Rhodes rapid and sustained learning. Am J Surg 2003;185:516-20.

8. Association of American Medical Colleges. Effective use of educational technology in medical education colloquium on educational technology: recommendations and guidelines for medical educators. AAMC Institute for Improving Medical Education. March 2007. Available from: https://members.aamc.org/eweb/upload/Effective\%20U se\%20of\%20Educational.pdf

9. Williams MJ, Lockey AS, Culshaw MC. Improved trauma management with advanced trauma life support (ATLS) training. J Accid Emerg Med 1997;14:81-3.

10. LeFlore JL, Anderson M, Michael JL, et al. Comparison of self-directed learning versus instructor-modeled learning during a simulated clinical experience. Simul Healthc 2007;2:170-7.

11. Rhodes M. Adult trauma resuscitation. In: Peitzman AB, Rhodes M, Schwab CW, et al, eds. The trauma manual: trauma and acute care surgery, $3^{\text {rd }}$ Ed. Philadelphia, PA: Lippincott Williams \& Wilkins: 2008. pp 71-80. 